Original Article

\title{
Impact of heart failure on functional recovery after hip fracture
}

\author{
Atsunori Itagaki, PT, MSc ${ }^{1,2)^{*}}$, Ayaka Kakizaki, PT ${ }^{3)}$, Miyuki Funahashi, PT $^{3)}$, \\ Kaori Sato, PT $^{3)}$, Kyoko Yasuhara, PT $^{3)}$, Akira Ishikawa, PT, MA, PhD ${ }^{4}$ ) \\ 1) Department of Cardiac Rehabilitation, The Cardiovascular Institute: 3-2-19 Nishiazabu, \\ Minato-ku, Tokyo, Japan \\ 2) Department of Internal Medicine and Rehabilitation Science, Tohoku University Graduate School of \\ Medicine, Japan \\ 3) Department of Rehabilitation, Fuyoukai Murakami Hospital, Japan \\ 4) Graduate School of Health Sciences, Hirosaki University, Japan
}

\begin{abstract}
Purpose] Heart failure has been identified as a risk factor for reduced physical function and falls; however, the impact of heart failure on functional recovery after a hip fracture is unclear. This study aimed to examine how heart failure and pre-fracture physical function affect recovery after a hip fracture. [Participants and Methods] The study population consisted of 122 patients with sub-acute hip fracture (mean age $81.7 \pm 9.7$ years, $18.9 \%$ male) who were divided into two groups: heart failure and non-heart failure. The outcome measurement was the functional independence measure effectiveness. A two-way analysis of variance was performed to investigate how heart failure and ambulatory ability prior to hip fracture were related to the functional independence measure effectiveness. [Results] Seventeen patients (13.9\%) had a history of heart failure. The two-way analysis of variance showed the two independent variables (heart failure and ambulatory ability before fracture) had significant main effects; however, their interaction effect was not significant. [Conclusion] Heart failure affects functional recovery after hip fracture independent of the pre-fracture physical function, and vice versa. Further research on rehabilitation in hip fracture patients with heart failure is required to develop strategies to overcome poor functional recovery in such patients.

Key words: Hip fracture, Heart failure, Functional recovery
\end{abstract}

(This article was submitted Nov. 2, 2018, and was accepted Dec. 19, 2018)

\section{INTRODUCTION}

The prevalence of heart failure (HF) greatly increases with age and is expected to increase even more as the population ages, referred to as the "HF pandemic"1), especially in an aging society such as the one in Japan ${ }^{2)}$. In HF patients, a loss of skeletal muscle mass and decreased bone mineral density are commonly observed ${ }^{3,4)}$, which contribute to reduced exercise capacity and a risk of falls with subsequent fractures. Indeed, HF has been already identified as a risk factor for fractures ${ }^{5,6)}$.

A reduced capacity for exercise prior to hospitalization is known to be a risk factor for hospitalization-associated disabilities $^{7)}$ and affects the functional recovery after a hip fracture ${ }^{8-10)}$, which is a major concern related to patient outcomes after such an incident. HF patients exhibit abnormal protein metabolism (accelerated catabolism and decreased assimilation) due to chronic inflammation, high oxidative stress, declining levels of anabolic hormones, and malnutrition, and conditions known as sarcopenia and cardiac cachexia ${ }^{3,11)}$. Thus, in addition to being associated with low physical function pre-fracture, HF may also contribute to poor functional recovery after a hip fracture independent of pre-fracture physical function.

*Corresponding author. Atsunori Itagaki (E-mail: atsunori_itagaki@med.tohoku.ac.jp)

(C2019 The Society of Physical Therapy Science. Published by IPEC Inc.

(c) (1) $\odot$ This is an open-access article distributed under the terms of the Creative Commons Attribution Non-Commercial No Derivatives

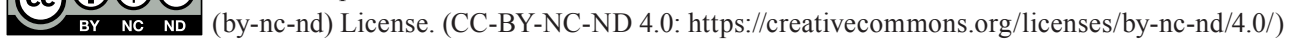


To the best of our knowledge, few studies have previously examined the postoperative functional recovery in hip fracture patients with HF, and little is known about how HF and pre-fracture physical function affect the patients' functional recovery after such an incident. We hypothesized that HF affects the patients' functional recovery after hip fracture independent of pre-fracture physical function, and vice versa.

The aim of this study was to investigate the influence of HF on functional recovery after a hip fracture and to verify the independence of its influence from preoperative physical function. To achieve this, we conducted a single center retrospective study of hip fracture patients with and without HF.

\section{PARTICIPANTS AND METHODS}

We retrospectively examined consecutive patients who underwent surgery for a hip fracture and were admitted to our rehabilitation unit between October 2014 and October 2017. The exclusion criteria were: 1) fractures that occurred during psychiatric hospital hospitalization, 2) transfer for reoperation, and 3) in-hospital death. In total, 122 patients were included in the study.

The study was conducted in accordance with all regulations of the Declaration of Helsinki. The study protocol was approved by the ethics committee of the Fuyoukai Murakami Hospital (No.2018-1-18).

Our hospital is a 120-bed convalescent rehabilitation hospital that adopts a multidisciplinary team approach. In the Japanese medical insurance system, patients were referred from acute hospitals, typically about 30 days after surgery for a hip fracture, and received hospital care in convalescent rehabilitation hospitals for up to 150 days after surgery. All patients in this study underwent rehabilitation programs on every day during hospitalization. Rehabilitation programs were provided by physical therapists and occupational therapists and consisted of a range of motion exercises, muscle strengthening exercises, gait exercises (using parallel bars, walkers, or canes as necessary), and ADL exercise. All patients were provided 60 to 180 -minute rehabilitation sessions per day.

All study variables were obtained from the patients' medical records. We investigated age, gender, body mass index (BMI; categorized as $<18.5, \geq 18.5$ to $<25.0$, and $\geq 25.0$, referring to recommended criteria for Asian populations by the World Health Organization ${ }^{12)}$ ), Charlson comorbidity index ${ }^{13)}$, fracture type [neck of femur and (inter) trochanteric], type of surgery (osteosynthesis and arthroplasty), the revised Hasegawa's dementia scale (HDS-R), the number of days from surgery to admission, Functional Independence Measure ${ }^{14)}$ (FIM), the length of hospital stay (LOS), ambulatory ability before fracture and at discharge [walking independently (outdoors), walking independently (indoors), and walking with assistance or wheelchair], and place of residence before fracture (own home, nursing home or residential home, and hospital). We also investigated the history of HF diagnosis.

Functional disability was assessed using the FIM, which is one of the most widely used assessment tools of ADL in patients with disability, at admission and discharge. FIM consists of 18 items divided into 6 subcategories: self-care (6 items), sphincter control (2 items), transfer ( 3 items), locomotion ( 2 items), communication ( 2 items), and social cognition ( 3 items). Each item is scored on a 7-point ordinal scale rating ranging from a score of 1 (total dependence) to a score of 7 (complete independence), and the total of the FIM scores range from 18 to 126. We used FIM effectiveness as the outcome measurement. FIM effectiveness can be calculated using the following formula: (FIM at discharge - FIM at admission) / (126 - FIM at admission). It is expressed as a percentage reflecting the proportion of potential improvement actually achieved during rehabilitation ${ }^{15,16)}$.

The participants were divided into two groups (the HF group and the non-HF group). Student's unpaired t-tests, $\chi^{2}$ tests, and one-way analysis of covariance (ANCOVA) were performed to compare the clinical characteristics between the two groups. Two-way analysis of variance (ANOVA) followed by Tukey's post-hoc-tests was performed to investigate how HF and ambulatory ability before fracture (independent variables) were related to the FIM effectiveness (dependent variable). Statistical significance was set at $\mathrm{p}<0.05$ for all analyses. Statistical analyses were performed using SPSS statistics version 22 (SPSS Inc., Chicago, IL, USA).

\section{RESULTS}

Of the 133 potential patients, 11 were excluded based on the criteria described above. Thus, 122 patients were included in the analysis. The mean [ \pm standard deviation (SD)] age of the patients was $81.7 \pm 9.7$ years and $18.9 \%$ of patients were male. Regarding the type of operation, $68.0 \%$ received osteosynthesis and $32.0 \%$ arthroplasty. Seventeen (13.9\%) of 122 patients had a history of HF diagnosis and were defined as the HF group. Compared with the non-HF group, the HF patients were significantly older $(\mathrm{p}=0.042)$ (Table 1$)$. The HF group patients also displayed lower FIM effectiveness during hospitalization even after adjustment for age, gender and ambulatory ability before fracture $(\mathrm{p}=0.048)$, despite there being no significant difference in FIM score between the groups at admission (Table 2A). The trajectories of change in ambulatory ability were similar between the two groups, but the rate of returning to the same residence as before the fracture was lower in the HF group ( $\mathrm{p}=0.039$ ) (Table $2 \mathrm{~B}$ ). The results of two-way ANOVA, with FIM effectiveness as the dependent variable, revealed that there were significant main effects of the two independent variables $[\mathrm{HF}(\mathrm{p}=0.026)$ and ambulation ability before fracture $(p<0.001)$, respectively], and that their interaction effect was not significant $(p=0.240)$. Tukey's post-hoc-test indicated that there were significant differences between walking independently (outdoors) vs. walking independently (indoors) $(\mathrm{p}=0.001)$, 
Table 1. Clinical characteristics of the HF group and non-HF group

\begin{tabular}{lccc}
\hline & $\begin{array}{c}\mathrm{HF} \\
(\mathrm{n}=17)\end{array}$ & $\begin{array}{c}\text { non-HF } \\
(\mathrm{n}=105)\end{array}$ & $\mathrm{p}$ value \\
\hline Age (years) & $84.9 \pm 5.9$ & $81.2 \pm 10.1$ & $\mathbf{0 . 0 4 2}$ \\
Gender (male, \%) & 8.7 & 15.2 & 0.421 \\
BMI & & & 0.162 \\
$\quad<18.5$ & 41.2 & 35.6 & \\
$\quad \geq 18.5$ to $<25.0$ & 35.3 & 54.8 & \\
$\quad \geq 25.0$ & 23.5 & 9.6 & \\
Charlson comorbidity index (\%) & & & 0.135 \\
$\quad 0-2$ & 52.9 & 76.2 & \\
$3-4$ & 41.2 & 21.0 & \\
$\quad$ F5 & 5.9 & 2.9 & \\
Fracture type (\%) & & & 0.659 \\
$\quad$ Neck of femur & 35.3 & 41.0 & \\
$\quad$ Inter) trochanteric & 64.7 & 59.0 & \\
Type of surgery (\%) & & & 0.808 \\
$\quad$ Osteosynthesis & 70.6 & 67.6 & \\
$\quad$ Arthroplasty & 29.4 & 32.4 & \\
HDS-R (points) & $18.8 \pm 9.0$ & $19.7 \pm 9.1$ & 0.889 \\
\hline
\end{tabular}

HF: heart failure; BMI: body mass index; HDS-R: revised Hasegawa's dementia scale.

$\mathrm{p}$ values less than 0.05 were written in bold.

Table 2. Differences in ADL recovery, length of hospital stay, change in ambulatory ability and change in place of residence between patients with and without HF

\begin{tabular}{|c|c|c|c|c|}
\hline \multirow[t]{2}{*}{$\mathrm{A}$} & \multirow{2}{*}{$\begin{array}{c}\mathrm{HF} \\
(\mathrm{n}=17)\end{array}$} & \multirow{2}{*}{$\begin{array}{l}\text { non-HF } \\
(\mathrm{n}=105)\end{array}$} & \multirow{2}{*}{$\begin{array}{c}\text { unpaired t-test } \\
\mathrm{p} \text { value }\end{array}$} & \multirow{2}{*}{$\begin{array}{c}\text { ANCOVA }^{\dagger} \\
\text { p value }\end{array}$} \\
\hline & & & & \\
\hline Number of days from surgery to admission (day) & $19.2 \pm 2.8$ & $20.4 \pm 5.8$ & 0.421 & 0.438 \\
\hline FIM at admission (points) & $58.5 \pm 27.7$ & $64.7 \pm 27.2$ & 0.383 & 0.712 \\
\hline FIM at discharge (points) & $76.7 \pm 38.5$ & $92.5 \pm 28.4$ & 0.046 & 0.066 \\
\hline FIM effectiveness $(\%)$ & $34.1 \pm 38.0$ & $51.6 \pm 28.7$ & 0.028 & 0.048 \\
\hline Length of hospital stay (day) & $63.8 \pm 28.2$ & $57.6 \pm 24.3$ & 0.341 & 0.404 \\
\hline
\end{tabular}

${ }^{\dagger}$ Adjusted for age, gender and ambulatory ability before fracture.

\begin{tabular}{lccc}
\hline B & HF & non-HF & $\chi^{2}$ test \\
\cline { 4 - 4 }$(\mathrm{n}=17)$ & & $\mathrm{p}$ value \\
\hline Ambulatory ability before fracture (\%) & & & 0.356 \\
$\quad$ Walking independently (outdoors) & 52.9 & 67.0 & \\
Walking independently (indoors) & 35.3 & 19.8 & \\
$\quad$ Walking with assistance or wheel chair & 11.8 & 13.2 & \\
Ambulatory ability at discharge (\%) & & & 0.503 \\
$\quad$ Walking independently (outdoors) & 5.9 & 16.0 & \\
$\quad$ Walking independently (indoors) & 35.3 & 35.8 & \\
$\quad$ Walking with assistance or wheel chair & 58.8 & 48.1 & \\
Place of residence before fracture (\%) & & & 0.655 \\
$\quad$ Own home & 64.7 & 73.6 & \\
$\quad$ Nursing home or residential home & 35.3 & 25.5 & \\
$\quad$ Hospital & 0 & 0.9 & \multirow{2}{*}{} \\
Discharge location (\%) & & & $\mathbf{0 . 0 3 9}$ \\
$\quad$ Same place of residence as prior to fracture & 58.8 & 81.1 & \\
$\quad$ Alternative location & & \\
\hline
\end{tabular}

HF: heart failure; ANCOVA: analysis of covariance.

$\mathrm{p}$ values less than 0.05 were written in bold.

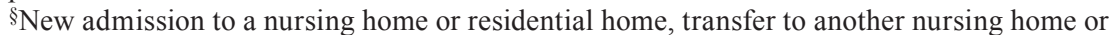

residential home or transfer to another hospital.

walking independently (outdoors) vs. walking with assistance or wheelchair $(\mathrm{p}=0.001)$ and walking independently (indoors) vs. walking with assistance or wheelchair $(\mathrm{p}=0.001)$ (Table 3). 
Table 3. The effects of heart failure and pre-fracture ambulatory status on functional recovery in hip fracture patients

\begin{tabular}{|c|c|c|c|c|c|}
\hline & \multicolumn{2}{|c|}{ FIM effectiveness (\%) } & \multicolumn{3}{|c|}{ Two-way ANOVA } \\
\hline & \multirow[b]{2}{*}{$\begin{array}{c}\mathrm{HF} \\
(\mathrm{n}=17)\end{array}$} & \multirow[b]{2}{*}{$\begin{array}{l}\text { non-HF } \\
(\mathrm{n}=105)\end{array}$} & \multicolumn{2}{|c|}{ Main effect $\mathrm{p}$ value } & \multirow[b]{2}{*}{ Interaction $\mathrm{p}$ value } \\
\hline & & & $(\mathrm{HF})$ & $\begin{array}{c}\text { (Ambulatory ability } \\
\text { before fracture) }\end{array}$ & \\
\hline \multicolumn{6}{|l|}{ Ambulatory ability before fracture } \\
\hline Walking independently (outdoors) & $57.4 \pm 16.2$ & $60.8 \pm 27.1$ & & & \\
\hline Walking independently (indoors) & $14.1 \pm 25.8$ & $35.9 \pm 21.1$ & 0.026 & $<0.001$ & 0.240 \\
\hline Walking with assistance or wheel chair & $3.0 \pm 1.4$ & $24.5 \pm 25.6$ & & & \\
\hline
\end{tabular}

ANOVA: analysis of variance; FIM: functional independence measure; HF: heart failure.

$\mathrm{p}$ values less than 0.05 were written in bold.

\section{DISCUSSION}

In this study, we hypothesized that HF affects the patients' functional recovery after a hip fracture independent of pre-fracture physical function and vice versa. To support this hypothesis, two-way ANOVA with FIM effectiveness as the dependent variable revealed that there were significant main effects of the two independent variables, HF and ambulation ability, prior to fracture. To the best of our knowledge, this is the first study to investigate the influence of HF on postoperative functional recovery after taking into consideration the pre-fracture physical function in hip fracture patients.

Several previous studies have already reported that low pre-fracture physical function is associated with poor functional recovery after a hip fracture ${ }^{8-10}$. Similarly, we confirmed that the influence of pre-fracture ambulatory ability on FIM effectiveness after a hip fracture is significant, and that this impact is independent of HF (Table 3). These results suggest that pre-fracture physical function plays an important role in predicting postoperative functional recovery in hip fracture patients.

On the other hand, very few studies have described the contribution of HF to functional recovery in hip fracture patients. Mathew and colleagues reported that HF was associated with an early delay in recovery of ADL after a hip fracture and took 12 months to recover to similar levels as non-HF patients ${ }^{17)}$. Our study results also suggested that HF was a risk factor for poor functional recovery after hip fracture, and we showed that this impact was independent of pre-fracture physical function. Hence, our study reveals new findings. Unfortunately, this mechanism cannot be explained because few studies have investigated the influence of HF on functional recovery after hip fracture; a possible explanation is that HF patients are in a state of chronic inflammation, high oxidative stress, declining anabolic hormones and malnutrition, with a high prevalence of protein metabolism and skeletal muscle abnormalities labelled as sarcopenia and cardiac cachexia ${ }^{3,11,18)}$. As with our results, although the prevalence of HF increases with age ${ }^{1)}$, these abnormalities are known to be age-independent. Therefore, the effect of exercise-based interventions on improving physical function may not have been as effective when compared with non-HF patients.

Recently, several studies have supported the influence of neuromuscular electrical stimulation therapy on improving physical function in HF patients, and its safety also has been confirmed ${ }^{19}, 20$ ). In addition, other studies have indicated a beneficial impact of branched-chain amino acid (BCAA) supplementation for HF patients ${ }^{21-24)}$, and a prospective study is currently being conducted to verify the combined effect of BCAA supplementation during conventional cardiac rehabilitation for Japanese HF patients ${ }^{25}$. Although these interventions are not routinely performed during rehabilitation of hip fracture patients, they may potentially be effective even for hip fracture patients with HF. Therefore, we emphasize the importance of further research on the utility of these additional interventions for functional recovery in hip fracture patients with HF.

We acknowledge some limitations of this study. First, we used categorized ambulatory ability [walking independently (outdoors), walking independently (indoors) and walking with assistance or wheelchair] as an indicator of physical function; but, as in the previous studies reporting the contribution of HF to decreased physical function ${ }^{26}$ ), continuous variables such as knee extension muscle strength or gait speed may be more sensitive for detecting the deterioration of physical function. However, when studying diseases resulting from unexpected injuries such as hip fracture, it may be difficult to obtain the continuous-variable physical functions before fracture. Second, we could not take into consideration the etiology of HF or duration between HF diagnosis and hip fracture occurrence. However, it may be difficult to obtain such detailed information about HF at convalescent rehabilitation hospitals like ours. Third, since this was a single-center study, this cohort may not be representative of all Japanese hip fracture patients and the results may therefore not be generalizable. The present study should be replicated using a larger number of patients selected from multiple, more diverse participating facilities.

In summary, $13.9 \%$ of patients had a history of HF diagnosis, and these patients showed poor functional recovery after hip fracture compared to those without HF. Furthermore, the influence of HF on functional recovery was independent of preoperative physical function. Recently, the utility of neuromuscular electrical stimulation therapy and BCAA supplementation for HF patients in improving physical function has been indicated in several studies, and we would like to emphasize the importance of confirming the effectiveness of these additional treatments in hip fracture patients with HF in a prospective intervention study. 


\section{Funding}

This research received no grant from any funding agency in the public, commercial or not-for-profit sectors.

Conflict of interest

The authors declare that there is no conflict of interest.

\section{ACKNOWLEDGEMENTS}

We would like to thank the all staff members of our hospital and Editage (www.editage.jp) for English language editing.

\section{REFERENCES}

1) Shimokawa H, Miura M, Nochioka K, et al.: Heart failure as a general pandemic in Asia. Eur J Heart Fail, 2015, 17: 884-892. [Medline] [CrossRef]

2) Konishi M, Ishida J, Springer J, et al.: Heart failure epidemiology and novel treatments in Japan: facts and numbers. ESC Heart Fail, 2016, 3: 145-151. [Medline] [CrossRef]

3) Collamati A, Marzetti E, Calvani R, et al.: Sarcopenia in heart failure: mechanisms and therapeutic strategies. J Geriatr Cardiol, 2016, 13: 615-624. [Medline]

4) Xing W, Lv X, Gao W, et al.: Bone mineral density in patients with chronic heart failure: a meta-analysis. Clin Interv Aging, 2018, 13: 343-353. [Medline] [CrossRef]

5) van Diepen S, Majumdar SR, Bakal JA, et al.: Heart failure is a risk factor for orthopedic fracture: a population-based analysis of 16,294 patients. Circulation, 2008, 118: 1946-1952. [Medline] [CrossRef]

6) Carbone L, Buzková P, Fink HA, et al.: Hip fractures and heart failure: findings from the cardiovascular health study. Eur Heart J, 2010, 31: 77-84. [Medline] [CrossRef]

7) Covinsky KE, Pierluissi E, Johnston CB: Hospitalization-associated disability: "She was probably able to ambulate, but I'm not sure". JAMA, 2011, 306: 1782-1793. [Medline] [CrossRef]

8) Cecchi F, Pancani S, Antonioli D, et al.: Predictors of recovering ambulation after hip fracture inpatient rehabilitation. BMC Geriatr, 2018, 18: 201. [Medline] [CrossRef]

9) Fitzgerald M, Blake C, Askin D, et al.: Mobility one week after a hip fracture—can it be predicted? Int J Orthop Trauma Nurs, 2018, 29: 3-9. [Medline] [CrossRef]

10) Pioli G, Lauretani F, Pellicciotti F, et al.: Modifiable and non-modifiable risk factors affecting walking recovery after hip fracture. Osteoporos Int, 2016, 27: 2009-2016. [Medline] [CrossRef]

11) Loncar G, Springer J, Anker M, et al.: Cardiac cachexia: hic et nunc. J Cachexia Sarcopenia Muscle, 2016, 7: 246-260. [Medline] [CrossRef]

12) Physical status: the use and interpretation of anthropometry. Report of a WHO Expert Committee. World Health Organ Tech Rep Ser, 1995, 854: 1-452. [Medline]

13) Charlson ME, Pompei P, Ales KL, et al.: A new method of classifying prognostic comorbidity in longitudinal studies: development and validation. J Chronic Dis, 1987, 40: 373-383. [Medline] [CrossRef]

14) Keith RA, Granger CV, Hamilton BB, et al.: The functional independence measure: a new tool for rehabilitation. Adv Clin Rehabil, 1987, 1: 6-18. [Medline]

15) Di Monaco M, Schintu S, Dotta M, et al.: Severity of unilateral spatial neglect is an independent predictor of functional outcome after acute inpatient rehabilitation in individuals with right hemispheric stroke. Arch Phys Med Rehabil, 2011, 92: 1250-1256. [Medline] [CrossRef]

16) Sánchez-Rodríguez D, Miralles R, Muniesa JM, et al.: Three measures of physical rehabilitation effectiveness in elderly patients: a prospective, longitudinal, comparative analysis. BMC Geriatr, 2015, 15: 142. [Medline] [CrossRef]

17) Mathew RO, Hsu WH, Young Y: Effect of comorbidity on functional recovery after hip fracture in the elderly. Am J Phys Med Rehabil, $2013,92: 686-696$. [Medline] [CrossRef]

18) Anker SD, Chua TP, Ponikowski P, et al.: Hormonal changes and catabolic/anabolic imbalance in chronic heart failure and their importance for cardiac cachexia. Circulation, 1997, 96: 526-534. [Medline] [CrossRef]

19) Ploesteanu RL, Nechita AC, Turcu D, et al.: Effects of neuromuscular electrical stimulation in patients with heart failure - review. J Med Life, 2018, 11: 107-118. [Medline]

20) Smart NA, Dieberg G, Giallauria F: Functional electrical stimulation for chronic heart failure: a meta-analysis. Int J Cardiol, 2013, 167: 80-86. [Medline] [CrossRef]

21) Sun H, Wang Y: Branched chain amino acid metabolic reprogramming in heart failure. Biochim Biophys Acta, 2016, 1862: 2270-2275. [Medline] [CrossRef]

22) Tsuji S, Koyama S, Taniguchi R, et al.: Nutritional status of outpatients with chronic stable heart failure based on serum amino acid concentration. J Cardiol, 2018, 72: 458-465. [Medline] [CrossRef]

23) Uchino $Y$, Watanabe M, Takata M, et al.: Effect of oral branched-chain amino acids on serum albumin concentration in heart failure patients with hypoalbuminemia: results of a preliminary study. Am J Cardiovasc Drugs, 2018, 18: 327-332. [Medline] [CrossRef]

24) Wandrag L, Brett SJ, Frost G, et al.: Impact of supplementation with amino acids or their metabolites on muscle wasting in patients with critical illness or other muscle wasting illness: a systematic review. J Hum Nutr Diet, 2015, 28: 313-330. [Medline] [CrossRef]

25) Takata M, Amiya E, Watanabe M, et al.: An exploratory study on the efficacy and safety of a BCAA preparation used in combination with cardiac rehabilitation for patients with chronic heart failure. BMC Cardiovasc Disord, 2017, 17: 205. [Medline] [CrossRef]

26) Sunnerhagen KS, Cider A, Schaufelberger M, et al.: Muscular performance in heart failure. J Card Fail, 1998, 4: 97-104. [Medline] [CrossRef] 